\title{
Association of genetic polymorphisms around the LIN28B gene and idiopathic central precocious puberty risks among Chinese girls
}

\author{
Zhijian $\mathrm{Hu}^{1}$, Ruimin Chen ${ }^{2}$ and Chunyan $\mathrm{Cai}^{2}$
}

BACKGROUND: Genome-wide association studies have identified rs314276, rs314263, rs7759938, and rs314280 in or near the LIN28B gene as associated with age at menarche. To date, the effect of polymorphisms in this gene on idiopathic central precocious puberty (ICPP) in Chinese girls have not been reported. The aim of this study was to evaluate the association of the four loci with ICPP in Chinese girls.

METHODS: In this study, we conducted a case-control study including 502 girls with ICPP and 489 controls. Four singlenucleotide polymorphisms (SNPs) were genotyped in both groups using an improved multiplex ligation detection reaction (iMLDR) technique.

RESULTS: Of the four SNPS of the LIN28B analyzed, three SNPS, rs314276, rs7759938, and rs314280, were associated with ICPP risk at $P<0.05$. The association of rs314276, however, was no longer significant after adjustment for multiple testing. Compared with rs7759938 TT or TC genotype, decreased ICPP risk was associated with CC (OR $=0.527,95 \% \mathrm{Cl}$ : 0.329-0.843) genotype $(P=0.008)$. Compared with rs314280 GG or GA genotype, decreased ICPP risk was associated with minor allele carrier $(A A)$ genotype $(O R=0.538,95 \% C l: 0.337-0.858, P=$ 0.009). The two identified variants showed the same association signals for ICPP.

CONCLUSION: In conclusion, common genetic variations (rs7759938 and rs314280) of LIN28B may contribute to ICPP susceptibility in Chinese girls.

$\mathrm{T}$ he onset of puberty is occuring earlier at least over the past several decades worldwide (1). Sexual development in nine reprentative cities between 2003 and 2005 in China, revealed a secular trend of earlier age of puberty onset $(2,3)$. The incidence of precocious puberty was 29 per 100,000 girls per year (4). In China, any signs of secondary sexual maturation below the population average ( $<8$ for girls or $<9$ for boys) are considered precocious puberty. The latter can be subdevided into central precocious puberty (CPP) which is initiated by activation of hypothalamic gonadotropin-releasing-hormone secretion and peripheral precocious puberty. Furthermore, 90 percent of CPP is idiopathic central precocious puberty (ICPP) and girls have a higher incidence of precocious puberty than boys, with a ratio of 20:1. Height in children with CPP may be attenuated due to the premature closure of the growth plates. In addition, the psychological development of children with CPP is not in synchrony with their development.

The onset of puberty is initiated by reemergence of pulsatile in both amplitude and frequency, gonadotropin-releasing hormone release from the hypothalamus but the mechanisms that activate the onset of puberty remain poorly understood. Besides environmental factors, genetic factors play an important role in the timing of puberty. Phillip (5) found a $27.5 \%$ prevalence of family history among 147 patients with idiopathic CPP, infering that puberty onset may be a process governed by genetics. Additionally, candidate genes may mediate the onset of puberty in familial CPP. The genetic underpinnings of puberty may be a result of multiple candidate genes. LIN28B, a human ortholog of lin28 of the nematode Caenorhatidis elegans, which regulates let-7 microRNAs (miRNAs) controlling the timing of developmental event (6). A gain-of-function allele of lin-28 retards development in the nematode Caenorhatidis elegans (7). Silveira-Neto identified a heterozygous $L I N 28 B$ variant P. H199R in a girl who developed CPP at 5.2 y old (8). Park SW reported that the Korea girls with non-AC haplotypes (LIN28B polymorphisms, rs4946651 and rs369065) tended to have earlier puberty onset (9). However, the mechanisms whereby of $L I N 28 B$ via let -7 miRNA regulate puberty onset are not yet clear.

Genome-wide association has successfully identified many single-nucleotide polymorphisms (SNPs), which are associated with complex diseases and certain human features. SNP is the third generation of genetic marker caused by single-nucleotide variation. Recently, variations in or near $L I N 28 B$ gene were reported to be associated with age at menarche by genomewide association studies. Studies have found many SNPs related to menarche, such as rs314276 (10), rs314263 $(11,12)$, and rs314280 $(13,14)$ in LIN28B gene. Further analysis by

'Department of Epidemiology and Health Statistics, Fujian Provincial Key Laboratory of Environment factors and Cancer, School of Public Health, Fujian Medical University, Fujian, China; '2Department of Endocrinology, Fuzhou Children's Hospital of Fujian, Teaching Hospital of Fujian Medical University, Fujian, China. Correspondence: Ruimin Chen (chenrm321@sina.com)

Received 22 March 2016; accepted 26 March 2016; advance online publication 15 June 2016. doi:10.1038/pr.2016.107 
meta-analysis, found that the another SNP rs7759938 $(15,16)$ near LIN28B gene has a robust association with menarche, the milestone for female sexual maturation. Hence, it is conceivable that menarche related SNPs may associate with ICPP. This study aimed to detect the relationship between menarche related SNPs and ICPP.

\section{RESULTS}

All girls with ICPP $(n=506)$ and the controls $(n=485)$ were of Han ethnicity and their ages at enrollment were 7.01 \pm 0.90 $($ mean + SD) and $7.05 \pm 1.13$ y old, respectively. Significant differences in height, weight, BMI, mother's age at menarche, physically active and sleeping time were observed between the two groups, whereas no significant differences were observed in other variables (Table 1).

The primary information and allele frequencies observed are listed in Table 2. The genotype distributions of the four tested SNPs were consistent with the Hardy-Weinberg equilibrium in the control group $(P>0.05)$. Associations with ICPP for these

Table 1. Comparions of cases and controls by demographic characteristics and selected variables

\begin{tabular}{lccc}
\hline & Cases (506) & Controls(485) & $P^{*}$ \\
\hline Age (years) & $7.01 \pm 0.90$ & $7.05 \pm 1.13$ & 0.540 \\
Height & $128.35 \pm 9.80$ & $119.90 \pm 9.95$ & $<0.001$ \\
Weight & $26.82 \pm 6.04$ & $22.43 \pm 5.51$ & $<0.001$ \\
BMI & $15.48 \pm 2.22$ & $16.08 \pm 2.06$ & $<0.001$ \\
Mother's age at menarche (years) & $13.69 \pm 1.37$ & $14.50 \pm 1.32$ & $<0.001$ \\
$\quad$ 13 & 201 & 97 & $<0.001$ \\
$>13$ & 305 & 388 & \\
Mother's age at first birth & $26.13 \pm 3.25$ & $26.10 \pm 3.69$ & 0.881 \\
Physically active (hours) & & & $<0.001$ \\
$<0.5$ & 79 & 60 & \\
$0.5-1$ & 276 & 214 & \\
$>1$ & 151 & 211 & \\
Sleeping time & & & \\
$<21$ & 209 & 253 & 0.003 \\
$21-22$ & 255 & 197 & \\
$>22$ & 41 & 35 & \\
\hline
\end{tabular}

*Continuous variable: mean values \pm SD, $P$ values (two-side) from Student $t$-tests; categorical variables: percentages, $P$ values (two-side) from $\chi^{2}$ tests. four SNPs are given in Table 3. Three SNPs (rs314276, rs7759938, rs314280) showed significant associations with ICPP in both additive models ( $\mathrm{OR}=0.81,0.81$ and $0.797 ; 95 \% \mathrm{CI}$ : 0.658-0.998, $0.657-0.998$, and $0.646-0.982 ; P=0.048,0.048$, and 0.033 , respectively) and recessive models $(\mathrm{OR}=0.616,0.527$ and 0.538 ; 95\% CI: 0.387-0.979, 0.329-0.843, and 0.337-0.858; $P=0.041$, 0.007 , and 0.009 , respectively) after adjusting for BMI, mother's age at menarche, mother's age at first birth, physically active, and sleeping time. However, the other SNP (rs314263) was not associated with ICPP regardless of genetic models $(P>0.05)$. A MeffSidak method evaluated the corrected statistical significance of these associations. After multiple comparison adjustment, only two SNPs (rs7759938, rs314280) still showed significant associations with ICPP in recessive models at $P<0.05$.

To determinate whether the effects of the three varinats (rs314276, rs7759938, rs314280) were independent or not, a stepwise and conditional logistic regression analysis was undertaken. The method incorporates BMI, mother's age at menarche, mother's age at first birth, physically active, and sleeping time in the model, to which the SNPs were added one by one in an iterative process. The results showed that only the rs7759938 SNP remained in the model at last step and showed the strongest significant association with ICPP in both additive and recessive models. Subsequent addition of rs314280 and rs314276 did not show significant improvement of the model, which is partially explained by high LD among of the three SNPs. Then, Conditional logistic regression analysis was performed to investigate the independent effects among these replicated SNPs with ICPP. The two SNPs, rs314280 and rs314276, were conditioned on the effect of the rs7759938 SNP. None of them remained significant after conditioning on the associated marker (rs7759938) regardless of genetic models. This suggests that not one of the 3 SNPs is exerting an independent effect. In other words, the rs7759938 SNP explains the all association signals with ICPP. The $P$ values of these SNPs before and after the effect of rs7759938 was adjusted for, are shown in Table 4.

Then, the association analysis for the variant rs7759938 was stratified by quartiles of age at precocious puberty onset. The results are presented in Table 5. In the upper middle quartile, the variant showed significant associations with ICPP in both additive models (OR $=0.72 ; 95 \% \mathrm{CI}$ : $0.52-0.98 ; P=0.043)$ and recessive models $(\mathrm{OR}=0.32 ; 95 \% \mathrm{CI}: 0.14-0.74 ; P=0.007)$. In the lower middle quartile, the variant showed significant associations with ICPP in additive models (OR $=0.64 ; 95 \% \mathrm{CI}$ :

Table 2. Primary information of genotyped SNPs

\begin{tabular}{|c|c|c|c|c|c|c|c|c|}
\hline \multirow[b]{2}{*}{ SNPs } & \multirow{2}{*}{$\begin{array}{l}\text { Cytological } \\
\text { location }\end{array}$} & \multirow[b]{2}{*}{ Position $^{a}$} & \multirow{2}{*}{$\begin{array}{l}\text { Nearby } \\
\text { genes }^{b}\end{array}$} & \multirow{2}{*}{$\begin{array}{l}\text { Major, minor } \\
\text { allele }\end{array}$} & \multirow{2}{*}{$\begin{array}{l}\text { Region of } \\
\text { the gene }\end{array}$} & \multicolumn{2}{|c|}{ 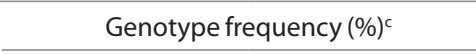 } & \multirow[b]{2}{*}{$H W E^{d}$} \\
\hline & & & & & & Case & Control & \\
\hline rs314276 & $6 q 21$ & 104960124 & LIN28B & $\mathrm{C}, \mathrm{A}$ & Intron & $49.2 / 412.9 / 8.89$ & $46.12 / 43.39 / 10.48$ & 0.899 \\
\hline rs7759938 & $6 q 21$ & 104931079 & LIN28B & $\mathrm{T}, \mathrm{C}$ & 3'UTR & $48.61 / 43.28 / 8.1$ & $46.67 / 42.5 / 10.83$ & 0.587 \\
\hline rs314280 & $6 q 21$ & 104952962 & LIN28B & $\mathrm{G}, \mathrm{A}$ & Intron & $48.62 / 43.08 / 8.3$ & $45.92 / 43.22 / 10.86$ & 0.752 \\
\hline
\end{tabular}

aPosition based on NCBI genome build 38 . ${ }^{b}$ Genes within $5 \mathrm{~kb}$ upstream and $5 \mathrm{~kb}$ downstream of the SNP. 'Genotype frequencies for AA (major allele homozygotes)/AB (heterozygotes)/BB (minor allele homozygotes). ${ }^{\mathrm{H}} \mathrm{HWE}$ (Hardy-Weinberg equilibrium) $P$ value in the control group.

SNP, single-nucleotide polymorphisms. 
Table 3. Selected SNPs and ICPP risk

\begin{tabular}{|c|c|c|c|c|c|c|c|c|c|}
\hline \multirow[b]{2}{*}{ SNPs } & \multicolumn{3}{|c|}{ ICPP risk, additive models ${ }^{\mathrm{a}}$} & \multicolumn{3}{|c|}{ Dominant models ${ }^{\mathrm{b}}$} & \multicolumn{3}{|c|}{ Recessive models ${ }^{c}$} \\
\hline & +B OR $(95 \% \mathrm{Cl})$ & $P$ & Adj- $P^{d}$ & AB/BB OR $(95 \% \mathrm{Cl})$ & $P$ & Adj- $P$ & BB OR $(95 \% \mathrm{Cl})$ & $P$ & Adj- $P$ \\
\hline rs314276 & $0.810(0.658-c$ & 8 & 060 & 325 & & 0.206 & $6(0.387-0$. & & 0.051 \\
\hline rs314263 & $900(0.738-1.096)$ & 295 & 0.356 & $925(0.704-1.21$ & ז & 0.662 & $760(0.507-1.140)$ & 0.1 & 0.227 \\
\hline rs7759938 & $0.810(0.657-0.998)$ & 048 & 06 & $2-1.1$ & 0 & 0.376 & $329-0.843)$ & 0.008 & 0.010 \\
\hline rs314280 & $797(0.646-0.982)$ & .033 & . & $0.840(0.640-1.104)$ & 0.211 & 0.23 & $0.538(0.337-0.858)$ & 0.009 & 0.011 \\
\hline \multicolumn{10}{|c|}{ 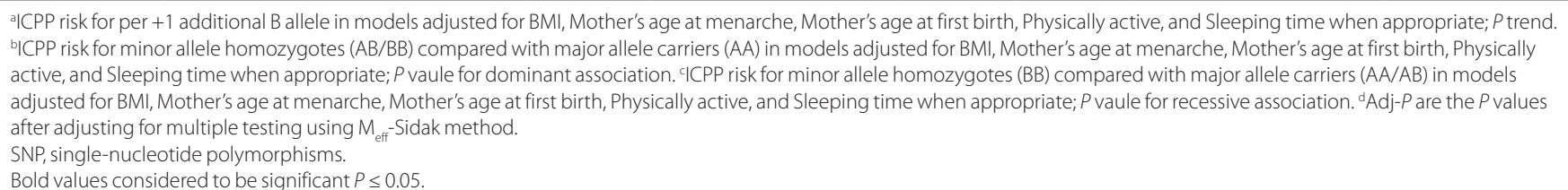 } \\
\hline
\end{tabular}

Table 4. Conditonal logistic regression analysis for independent effects among the identified SNPs in LIN28B

\begin{tabular}{|c|c|c|c|}
\hline Genetic model & SNPS & $\begin{array}{c}\text { Unconditional } \\
P \text { value }\end{array}$ & $\begin{array}{c}P \text { value conditional } \\
\text { on rs } 7759938\end{array}$ \\
\hline \multicolumn{4}{|l|}{ Additive models $\mathrm{s}^{\mathrm{a}}$} \\
\hline & rs314276 & 0.048 & 0.814 \\
\hline & rs314280 & 0.033 & 0.292 \\
\hline \multicolumn{4}{|c|}{ Dominant models ${ }^{\mathrm{b}}$} \\
\hline & rs314276 & 0.167 & 0.115 \\
\hline & rs314280 & 0.211 & 0.206 \\
\hline \multicolumn{4}{|c|}{ Recessive modelsc } \\
\hline & rs314276 & 0.041 & 0.121 \\
\hline & rs314280 & 0.009 & 1.000 \\
\hline \multicolumn{4}{|c|}{$\begin{array}{l}\text { alCPP risk for per }+1 \text { additional B allele in models adjusted for BMI, Mother's age at } \\
\text { menarche, Mother's age at first birth, Physically active, and Sleeping time when } \\
\text { appropriate; } P \text { trend. } \text {. ICPP risk for minor allele homozygotes (AB/BB) compared } \\
\text { with major allele carriers (AA) in models adjusted for BMI, Mother's age at menarche, } \\
\text { Mother's age at first birth, Physically active, and Sleeping time when appropriate; } \\
P \text { vaule for dominant association. 'ICPP risk for minor allele homozygotes (BB) } \\
\text { compared with major allele carriers (AA/AB) in models adjusted for BMI, Mother's age } \\
\text { at menarche, Mother's age at first birth, Physically active, and Sleeping time when } \\
\text { appropriate; } P \text { vaule for recessive association. } \\
\text { SNP, single-nucleotide polymorphisms. } \\
\text { Bold values considered to be significant } P \leq 0.05 \text {. }\end{array}$} \\
\hline
\end{tabular}

0.46-0.89; $P=0.007)$, dominant models $(\mathrm{OR}=0.61 ; 95 \% \mathrm{CI}$ : $0.40-0.92 ; P=0.02)$ and recessive models $(\mathrm{OR}=0.44 ; 95 \% \mathrm{CI}$ : $0.20-0.96 ; P=0.007)$. However, in the lower quartile or upper quartile, the variant was not associated with ICPP regardless of genetic models $(P>0.05)$. The results indicated that the variants may influence "mild" ICPP but not extremely early or later ICPP.

\section{DISCUSSION}

In this hospital-based study conducted among Han Chinese girls, the association of four SNPs around LIN28B previously associated with age of menarche was evaluated. We found that two SNPs (rs7759938 and rs314280) in the LIN28B gene were associated with the risk of ICPP. Neither of these polymorphisms has been previously found to be associated with ICPP susceptibility among Han Chinese girls.

LIN28B may serve as a trigger for menarche and act as a switch for the timing of puberty. LIN28B activity is dysregulated through various mechanisms in pubertal development, and it was found to be crucial for early onset and progression of puberty. A recent study in mice showed that genetic modification of the Lin28-Let-7 pathway by over-expression of Lin $28 B$ increased body size and delayed the onset of puberty (17). Therefore, common genetic variation in this key gene may underscore the pathogenesis of ICPP. Furthermore, LIN28B polymorphisms have been previously evaluated in relation to central precocious puberty and early puberty risk in Korea girls. Carriers of the non-AC haplotypes of LIN28B (rs4946651 and rs369065) have been reported to have significantly increased the risk of central precocious puberty in Korea girls (9). Our analysis similarly found that girls with LIN28B rs7759938 C allele or rs314280 A allele decreased the ICPP risk, especially under the recessive model. These variants rs7759938 and rs314280 of LIN28B have previously been identified as relevant for adult height $(7,18)$ and timing of pubertal onset (10,12,19-22). Another LIN28B SNP (rs314276) was reached a normal significance level (Ptrend $=0.048)$. Longitudinal analyses revealed rs314276 in LIN28B was related to increase body weight and BMI (23). This association, however, was no longer statistically significant after adjustment for multiple comparisons.

The independent effects for the three variants in LIN28B were evaluated through modeling by both stepwise and conditional logistic regression. Based on our results, the two SNPs, rs314280, rs314276, showed no significant relationship with ICPP while the effect of rs7553685 was adjusted, demonstrating no further independent signals of association with ICPP for the two variants. This suggested that the three variants have high LD between each other, and rs7553685 can represent the all association signals with ICPP for the three SNPs. A multiple computational analysis assessed the set SNPs for potential functional effect by using three computational tools: SNP Function Prediction (24), F-SNP (25), and SNP Function Portal (26), which are the function analysis and selection tools for single nucleotide polymorphisms. In this study, two of the three identified LIN28B variants, rs314276 and rs314280, were predicted to be possible transcription factor binding sites from all of the web servers. Because it is also plausible that these SNPs are not functional polymorphisms but are in tight LD with functional polymorphisms, these SNPs may possibly influence gene expression level or pre-mRNA splicing, or are 
Articles | Huetal.

Table 5. The variant (rs7759938) and ICPP risk with different onset ages of precocious puberty

\begin{tabular}{|c|c|c|c|c|c|c|}
\hline \multirow[b]{2}{*}{ Onset ages ${ }^{\mathrm{a}}$} & \multicolumn{2}{|c|}{ ICPP risk, additive models ${ }^{\mathrm{b}}$} & \multicolumn{2}{|c|}{ Dominant models ${ }^{c}$} & \multicolumn{2}{|c|}{ Recessive models $^{d}$} \\
\hline & +B OR $(95 \% \mathrm{Cl})$ & $P$ & AB/BB OR $(95 \% \mathrm{Cl})$ & $P$ & BB OR $(95 \% \mathrm{Cl})$ & $P$ \\
\hline$<6.5$ & $0.92(0.68-1.25)$ & 0.601 & $1.02(0.67-1.53)$ & 0.936 & $0.67(0.33-1.30)$ & 0.226 \\
\hline $6.5-7.2$ & $0.64(0.46-0.89)$ & 0.007 & $0.61(0.40-0.92)$ & 0.020 & $0.44(0.20-0.96)$ & 0.041 \\
\hline $7.2-7.7$ & $0.72(0.52-0.98)$ & 0.043 & $0.81(0.53-1.22)$ & 0.308 & $0.32(0.14-0.74)$ & 0.007 \\
\hline$>7.7$ & $0.84(0.62-1.14)$ & 0.266 & $0.91(0.61-1.37)$ & 0.655 & $0.55(0.27-1.13)$ & 0.101 \\
\hline
\end{tabular}

${ }^{a}$ Quartiles for age at precocious puberty onset. Lower quartile $=6.5$, median=7.2, upper quartile=7.7. ${ }^{\circ} \mathrm{CPP}$ risk for per +1 additional B allele in models adjusted for BMI, Mother's age at menarche, Mother's age at first birth, Physically active, and Sleeping time when appropriate; $P$ trend. ᄃICPP risk for minor allele homozygotes (AB/BB) compared with major allele carriers (AA) in models adjusted for BMI, Mother's age at menarche, Mother's age at first birth, Physically active, and Sleeping time when appropriate; $P$ vaule for dominant association. 'ICPP risk for minor allele homozygotes(BB) compared with major allele carriers (AA/AB) in models adjusted for BMI, Mother's age at menarche, Mother's age at first birth, Physically active, and Sleeping time when appropriate; $P$ vaule for recessive association.

Bold values considered to be significant $P \leq 0.05$

in LD with ungenotyped variants that do. Therefore, an etiologic role of these two SNPs in the LIN28B gene in the pathogenesis of ICPP warrants further study.

Among girls from Taiwan, rs314280 polymorphism was not a useful marker for prediction of the susceptibility of precocious puberty in a small case-control study (50 cases and 45 controls) (27). This result is not consistent with our study and other previous studies $(11,12,21,28)$. The discrepancy may be due to different illness classifications, sample size, confounding factors, racial and disease variations. The other SNP rs314263 in LIN28B in this study showed no significant association with ICPP. Although other reports have shown links with SNP and menarche (11), no previous studies have confirmed an association with ICPP for this variant.

The current study has several strengths. Most importantly, extensive information on anthropometrics and lifestyle factors were collected in this study to adjust for confounding factors. Additionally, Chinese women living in Fujian are relatively homogeneous in ethnic background, all of whom are classified into a single ethnic group (Han Chinese). Therefore, a potential confounding effect by ethnicity is of limited concern.

In conclusion, our study has found evidence that two SNPs rs7759938 and rs314280 of LIN28B were associated with ICPP in Han Chinese girls. Further functional researches and multiple-center studies with large-scale samples are warranted to confirm our findings.

\section{METHODS}

\section{Study Population}

This study was conducted as a hospital-based case-control design involving 991 unrelated individuals enrolled to the Fuzhou Children's Hospital of Fujian. From 2010 to 2013, incident cases of ICPP were recruited and the diagnosis of central precocious puberty followed the criteria of the Lawson Wilkins Pediatric Endocrine Society. Controls were selected from the same hospital as the patients in all centers, except in the Pediatric endocrinology clinic center. Controls were matched to cases by age, sex, ethnicity, and geographic location of origin. The study included 502 patients and 489 controls. All participants were asked to donate a blood sample for DNA extraction, and underwent a personal interview for socio-demographic information, familial and medical history, diet and living habits. Standing height without shoes was measured using a height measuring instrument to the nearest $0.1 \mathrm{~cm}$. Body weight was measured with the lightest clothing to the nearest $0.1 \mathrm{~kg}$ by an electronic weighing scale. Written informed consent was obtained from the parents of the participants prior to enrollment. This study was reviewed and approved by the
Ethics Committee of Fuzhou Children's Hospital of Fujian, and was conducted in agreement with the Declaration of Helsinki Principles.

\section{SNP Selection and Genotyping}

After a review of the medical literature (10-16), four significant SNPs were selected namely, rs314276, rs314263, rs7759938, rs314280 around LIN28B. All SNPs selected were based on the following: (i) a Chinese population frequency, based on the minor allele frequency, of $>5 \%$; (ii) previously described significant association with menarche $\left(P<5 \times 10^{-8}\right)$; (iii) the SNPs were located in the LIN28B gene or within the $5-\mathrm{kb}$ region flanking the gene.

Genomic DNA from the blood samples was extracted using a protease K digestion (Beyotime, Shanghai, China) and phenol-chloroform (Junrui, Shanghai, China) extraction and purification according to a standard procedure. The genomic DNA was then stored at -20 ${ }^{\circ} \mathrm{C}$. Thereafter, DNA samples were subjected to SNP genotyping. The genotyping of SNPs were carried out by Shanghai Genesky Bio-Tech (Genesky, Shanghai, China), using an improved multiplex ligase detection reaction method. Briefly, a multiplex polymerase chain reaction was designed to amplify the four SNP loci. Then, the ligation reaction was performed and the raw data were analyzed by Gene Mapper 4.1 (Applied Biosystems, CA). All of the primers and probes (Sangon, Shanghai, China) are listed in Supplementary Table S1 online. Genotyping was performed in a blinded fashion without knowledge of the patients' clinical data, and approximately $10 \%$ of the samples were randomly repeated for quality control purposes. Genotyping call rates were $94 \%$ and the concordance rate reached $99.5 \%$.

\section{Statistical Analysis}

Pearson $\chi^{2}$ and unpaired Student's $t$-test examined the differences between ICPP patients and controls for categorical and continuous variables, respectively. Testing for deviations from Hardy-Weinberg equilibrium was carried out using a Pearson goodness-of-fit test. Each genotype of examined polymorphisms was assessed by unconditional logistic regression analyses under the log-additive (major allele homozygotes vs. heterozygotes vs. minor allele homozygotes) models, dominant (major allele homozygotes vs. heterozygotes + minor allele homozygotes), recessive (major allele homozygotes + heterozygotes vs. minor allele homozygotes) models of inheritance after adjusting for confounding factors, respectively. $P$ values in models were adjusted for multiple comparisons using $\mathrm{M}_{\text {eff }}$-Sidak method (29), to reduce falsepositive rates. Conditional and stepwise logistic regression was used to determine whether independent effects of SNPs exsited.

The majority of statistical analyses were conducted with SAS v9.2 (StataCorp LP, College Station, TX). All statistical tests were twotailed, and $P \leq 0.05$ values were interpreted as statistically significant.

\section{Power Calculations}

Power for association studies depends on the sample size, the specified level of type I error, and the genotype frequencies in patients and control subjects. Genotype frequencies depend on the population frequency of the allele of interest and its effect on disease risk. "Power for Genetic Association analyses" package (30) was used to estimate the statistical power for statistically significant results concerning individual polymorphism data. Under a recessive model, results showed a 
power of $>80 \%$ for the significant association of the variants with ICPP for the allele frequencies and sample size in the present study.

\section{SUPPLEMENTARY MATERIAL}

Supplementary material is linked to the online version of the paper at http:// www.nature.com/pr

\section{STATEMENT OF FINANCIAL SUPPORT}

This research was supported by grants: the Natural Science Foundation of Fujian Province, China (Grant No.2009J01321) and the Science and Technology Project of Fuzhou Health System, China (Grant No.2013-S-wt6)

Disclosure: The authors have no financial relationships relevant to this article to disclose.

\section{REFERENCES}

1. Parent AS, Rasier G, Gerard A, et al. Early onset of puberty: tracking genetic and environmental factors. Horm Res 2005;64 Suppl 2:41-7.

2. Ma HM, Du ML, Luo XP, et al.; Pubertal Study Group of the Society of Pediatric Endocrinology and Genetic Disease, Chinese Medical Association. Onset of breast and pubic hair development and menses in urban chinese girls. Pediatrics 2009;124:e269-77.

3. Ma HM, Chen SK, Chen RM, et al.; Pubertal Study Group of the Society of Pediatric Endocrinology and Genetic Disease, Chinese Medical Association. Pubertal development timing in urban Chinese boys. Int J Androl 2011;34(5 Pt 2):e435-45.

4. Fuqua JS. Treatment and outcomes of precocious puberty: an update. J Clin Endocrinol Metab 2013;98:2198-207.

5. Phillip M, Lazar L. Precocious puberty: growth and genetics. Horm Res 2005;64 Suppl 2:56-61.

6. Viswanathan SR, Daley GQ, Gregory RI. Selective blockade of microRNA processing by Lin28. Science 2008;320:97-100.

7. Tommiska J, Wehkalampi K, Vaaralahti K, Laitinen EM, Raivio T, Dunkel L. LIN28B in constitutional delay of growth and puberty. J Clin Endocrinol Metab 2010;95:3063-6.

8. Silveira-Neto AP, Leal LF, Emerman AB, et al. Absence of functional LIN28B mutations in a large cohort of patients with idiopathic central precocious puberty. Horm Res Paediatr 2012;78:144-50.

9. Park SW, Lee ST, Sohn YB, et al. LIN28B polymorphisms are associated with central precocious puberty and early puberty in girls. Korean J Pediatr 2012;55:388-92.

10. Ong KK, Elks CE, Li S, et al. Genetic variation in LIN28B is associated with the timing of puberty. Nat Genet 2009;41:729-33.

11. He C, Kraft P, Chen C, et al. Genome-wide association studies identify loci associated with age at menarche and age at natural menopause. Nat Genet 2009;41:724-8.

12. Tanikawa C, Okada Y, Takahashi A, et al. Genome wide association study of age at menarche in the Japanese population. PLoS One 2013;8:e63821.

13. Sulem P, Gudbjartsson DF, Rafnar T, et al. Genome-wide association study identifies sequence variants on $6 \mathrm{q} 21$ associated with age at menarche. Nat Genet 2009;41:734-8.
14. Carty CL, Spencer KL, Setiawan VW, et al. Replication of genetic loci for ages at menarche and menopause in the multi-ethnic Population Architecture using Genomics and Epidemiology (PAGE) study. Hum Reprod 2013;28:1695-706.

15. Elks CE, Perry JR, Sulem P, et al.; GIANT Consortium. Thirty new loci for age at menarche identified by a meta-analysis of genome-wide association studies. Nat Genet 2010;42:1077-85.

16. Croteau-Chonka DC, Lange LA, Lee NR, Adair LS, Mohlke KL. Replication of LIN28B SNP association with age of menarche in young Filipino women. Pediatr Obes 2013;8:e50-3.

17. Zhu H, Shah S, Shyh-Chang N, et al. Lin28a transgenic mice manifest size and puberty phenotypes identified in human genetic association studies. Nat Genet 2010;42:626-30.

18. Lango Allen H, Estrada K, Lettre G, et al. Hundreds of variants clustered in genomic loci and biological pathways affect human height. Nature 2010; 467:832-8

19. Widén E, Ripatti S, Cousminer DL, et al. Distinct variants at LIN28B influence growth in height from birth to adulthood. Am J Hum Genet 2010;86:773-82.

20. Perry JR, Stolk L, Franceschini N, et al. Meta-analysis of genome-wide association data identifies two loci influencing age at menarche. Nat Genet 2009;41:648-50.

21. Leinonen JT, Surakka I, Havulinna AS, et al. Association of LIN28B with adult adiposity-related traits in females. PLoS One 2012;7:e48785.

22. Delahanty RJ, Beeghly-Fadiel A, Long JR, et al. Evaluation of GWAS-identified genetic variants for age at menarche among Chinese women. Hum Reprod 2013;28:1135-43.

23. Ong KK, Elks CE, Wills AK, et al. Associations between the pubertal timing-related variant in LIN28B and BMI vary across the life course. J Clin Endocrinol Metab 2011;96:E125-9.

24. Yuan HY, Chiou JJ, Tseng WH, et al. FASTSNP: an always up-to-date and extendable service for SNP function analysis and prioritization. Nucleic Acids Res 2006;34(Web Server issue):W635-41.

25. Lee PH, Shatkay H. F-SNP: computationally predicted functional SNPs for disease association studies. Nucleic Acids Res 2008;36(Database issue):D820-4.

26. Wang P, Dai M, Xuan W, et al. SNP Function Portal: a web database for exploring the function implication of SNP alleles. Bioinformatics 2006;22:e523-9.

27. Chou IC, Wang CH, Lin WD, Tsai CH, Tsai FJ. Association study in Taiwanese girls with precocious puberty. J Pediatr Endocrinol Metab 2011;24:103-4.

28. Perry JR, Stolk L, Franceschini N, et al. Meta-analysis of genome-wide association data identifies two loci influencing age at menarche. Nat Genet 2009;41:648-50.

29. Nyholt DR. A simple correction for multiple testing for single-nucleotide polymorphisms in linkage disequilibrium with each other. Am J Hum Genet 2004;74:765-9.

30. Menashe I, Rosenberg PS, Chen BE. PGA: power calculator for case-control genetic association analyses. BMC Genet. 2008;9:36-41. 